\title{
Exogenous hydrogen sulfide alleviates surgery-induced neuroinflammatory cognitive impairment in adult mice by inhibiting NO signaling
}

Lijun Yin ${ }^{\dagger}$, Shunli Gao and Changkun Li ${ }^{*}$

\begin{abstract}
Background: To investigate the effect and mechanisms of exogenous hydrogen sulfide in surgery-induced neuroinflammatory cognitive dysfunction.

Methods: C57BL/6 J male mice ( $n=140)$ were used and randomly divided into seven groups: the sham group, surgery group, GYY4137 group, L-NAME group, surgery+GYY4137 group, surgery +L-NAME group, and surgery+GYY4137 + L-NAME group. After the interventions, open field tests (OFT) and the Morris water maze (MWM) test were conducted to evaluate learning and memory abilities in the mice. ELISAs, nitrate reductase assays, and Western blots (WB) were conducted to evaluate interleukin-1 beta (IL-1B), tumor necrosis factor-alpha (TNF-a), nitric oxide (NO), inducible nitric oxide synthase (iNOS), malondialdehyde (MDA), and antioxidant enzyme superoxide dismutase (SOD) levels. Furthermore, the expression level of microglial marker ionized calcium binding adaptor molecule 1 (IBA) in the hippocampal CA1 and CA3 areas was detected by an immunohistochemical (IHC) assay and apoptotic cells were observed using terminal deoxynucleotidyl transferase dUTP end-labeling (TUNEL) staining kits.

Results: We found that surgery induced neuroinflammatory cognitive dysfunction, oxidative stress, microglial activation, and cell apoptosis in the hippocampus. Moreover, following surgery, NO and iNOS levels were elevated in the hippocampus. Notably, all the effects caused by surgery were reversed by the $\mathrm{H}_{2} \mathrm{~S}$ donor GYY4137 or the iNOS inhibitor N(gamma)-nitro-L-arginine methyl ester (L-NAME). However, the combined application of GYY4137 and L-NAME was not superior to treatment with either agent alone and the effect of GYY4137 was similar to that of L-NAME.

Conclusion: The long-acting hydrogen sulfide donor GYY4137 had an ability to reversed the cognitive deficits and inflammation caused by carotid artery exposure surgery. This implies that NO signaling pathways might participate in this process. These results indicate that exogenous $\mathrm{H}_{2} \mathrm{~S}$ may be a promising therapy for POCD.
\end{abstract}

Keywords: POCD, Neuroinflammation, $\mathrm{H}_{2} \mathrm{~S}$, iNOS, NO

\footnotetext{
* Correspondence: lichangkunmr@163.com

${ }^{\dagger}$ Lijun Yin and Shunli Gao contributed to the work equally and should be regarded as co-first authors.

Department of Anesthesiology, Baodi Clinical College of Tianjin Medical University, No.8 Guangchuan Road, Baodi District, Tianjin 301800, China
}

(c) The Author(s). 2020 Open Access This article is distributed under the terms of the Creative Commons Attribution 4.0 International License (http://creativecommons.org/licenses/by/4.0/), which permits unrestricted use, distribution, and reproduction in any medium, provided you give appropriate credit to the original author(s) and the source, provide a link to the Creative Commons license, and indicate if changes were made. The Creative Commons Public Domain Dedication waiver (http://creativecommons.org/publicdomain/zero/1.0/) applies to the data made available in this article, unless otherwise stated. 


\section{Background}

Post-operative cognitive dysfunction (POCD) is characterized by cognitive impairments, including learning and memory deficits in patients after anesthesia and surgery, and affects about $30 \%$ of young and elderly patients after hospital discharge [1-3]. In addition to negative factors such as impaired communication and reduced daily activities, life quality, and work performance, POCD is related to higher morbidity and mortality, longer hospitalization, and greater economic spending [3-6]. Aging, education level, surgical trauma, anesthesia, postoperative analgesia, and infection are risk factors for developing POCD [7-9]. Notably, structural and biochemical alterations, such as reduced neurogenesis and the induction of neuroinflammation in the hippocampus, an area of the brain mainly responsible for cognition that is highly vulnerable to aging, are most likely the mechanisms underlying POCD [10-13]. Animal studies suggest that neuroinflammation may be a major cause of anesthesia and surgery-induced cognitive impairment $[14,15]$. Nevertheless, the causes and pathogenesis of POCD are yet to be fully investigated.

Nitric oxide (NO) plays a crucial role in supporting normal physiological functions, but pathological conditions such as inflammation can stimulate the production of high concentrations of NO, which may initiate neurodegeneration [16]. Moreover, NO is involved in cellular modifications such as microglial activation, neuronal cell apoptosis, and oxidative stress, and overproduction of NO impairs cognitive function. The bioavailability of NO is recognized as a predictive risk factor for Alzheimer's disease (AD) and early POCD [17-19]. NO is synthetized by nitric oxide synthases (NOS), which are grouped into three classes: endothelial NOS (eNOS), neuronal NOS (nNOS), and inducible NOS (iNOS). eNOS and nNOS are associated with endothelial and neuronal cells, respectively, and produce low and transient concentrations of NO. The production of iNOS is not restricted to specific cells but is expressed following different stimuli such as inflammation, and is a major pro-inflammatory and destructive mediator in inflammatory disease [20]. iNOS also promotes synaptic plasticity and brain function deficits, such as cognitive deficits $[17$, 21]. L-nitro-arginine methyl ester (L-NAME), an inhibitor of NOS that can inhibit NO biosynthesis, can attenuate brain dysfunction [22]. Therefore, NO may represent a pathogenic signal for POCD.

Hydrogen sulfide $\left(\mathrm{H}_{2} \mathrm{~S}\right)$, the third gaseous transmitter alongside $\mathrm{NO}$, can regulate the release of $\mathrm{NO}$ and its interaction with $\mathrm{NO}$ in the hippocampus can alleviate brain dysfunction [22-24]. In addition, $\mathrm{H}_{2} \mathrm{~S}$ exerts both pro- and anti-inflammatory effects [24]. The exogenous hydrogen sulfide donor GYY4137 induces the slow release of $\mathrm{H}_{2} \mathrm{~S}$ and reduces intestinal inflammation and protects against intestinal ischemia via eNOS-dependent pathways [25].

In the present work, we investigated the effect and mechanism of action of the $\mathrm{H}_{2} \mathrm{~S}$ donor GYY4137 in a surgery-induced neuroinflammatory cognitive deficit model in adult mice.

\section{Methods}

\section{Animals and groups}

We used 140 healthy 8-week-old adult male C57BL/6 J mice weighing 25-30 g, purchased from the Animal Experiment Center of the Institute of Radiation Medicine of the Chinese Academy of Medical Science. All mice were maintained for at least 1 week in a temperaturecontrolled room at $25^{\circ} \mathrm{C}$ and $60 \%$ relative humidity with a $12 \mathrm{~h}$ light cycle and given free access to a standard laboratory diet and water before experiments. The mice were treated according to the ethical guidelines of the Animal Experiment Center of the Institute of Radiation Medicine of the Chinese Academy of Medical Science, and the Animal Studies Committee of the Animal Experiment Center of the Institute of Radiation Medicine of the Chinese Academy of Medical Science approved the experimental protocol. Mice were randomly divided into seven groups: sham group (all surgical procedures were performed without right carotid artery exposure on Day 0), surgery group (right carotid artery exposure on Day 0), GYY4137 group (intraperitoneal injection of 50 $\mathrm{mg} / \mathrm{kg}$ GYY4137 in phosphate-buffered saline (PBS) $1 \mathrm{~h}$ before sham surgery), L-NAME group (water intake with $15 \mathrm{mg} / \mathrm{kg}$ L-NAME in the drinking water from Day 1 before sham surgery to Day 3 post-surgery), surgery+GYY4137 group (intraperitoneal injection of $50 \mathrm{mg} / \mathrm{kg}$ GYY4137 $1 \mathrm{~h}$ before carotid artery exposure), surgery+LNAME group (water intake with $15 \mathrm{mg} / \mathrm{kg}$ of L-NAME before carotid artery exposure up to Day 3 postsurgery), and surgery+GYY4137 + L-NAME group. Each group included 20 mice.

\section{Right carotid artery exposure-induced surgical inflammatory injury model}

After mice were anesthetized with 3\% sevoflurane, a right carotid artery exposure surgery procedure was carried out, as previously described [1, 26]. After about 10 min of the total surgical procedure was performed, all animals received compound lidocaine cream containing $25 \mathrm{mg}$ prilocaine and $25 \mathrm{mg}$ lidocaine (Beijing Ziguang Medication Manufacture Corporation Ltd., China) on the wound.

\section{Open field test (OFT)}

On the second day post-surgery, the OFT was performed to determine the spontaneous locomotor activity of mice, as previously described [26]. Briefly, a plastic 
open field box $(50 \times 50 \times 23 \mathrm{~cm})$ with 16 equal sector bases was used. Mice were placed at the corner for 5 min of acclimatization, and in the next $5 \mathrm{~min}$, while all four paws of the animals were placed in a new square, the number of crossings were recorded and raising of the forepaws was recorded as the number of rears. During the test procedure, 5\% ethyl alcohol was employed for cleaning the box to remove animal cues.

\section{Morris water maze (MWM)}

On Days 3-8 post-surgery, the MWM test was conducted to evaluate learning and memory ability. The experiment was performed using water maze equipment (Jiliang software (DigBehv) technology company, Shanghai, China) from 9:00-12:00 am every day over a total of 6 days, as previously described [26, 27]. The first 5 days were for directional navigation to test the learning ability of mice. Two indicators were recorded: one was the time it took the mouse to find the platform, named "Escape Latency," and the second was the distance it took to find the platform, named "Swimming Distance (Path Length)." When the MWM was performed, the mouse was placed into the pool facing the wall. Then, the time and distance were recorded from when/where the mouse was placed into the water until it found the platform. If more than 60 $s$ passed without it finding the platform, the mouse was guided to arrive to the platform and allowed to rest for 10 $\mathrm{s}$ on the platform and the escape latency was recorded as $60 \mathrm{~s}$. The memory ability of mice was tested on the last day using space exploration. The platform was removed, and the mouse was placed in the water twice facing to the pool wall. Then, the time and distance of swimming in the third quadrant (target quadrant) was recorded.

\section{Hippocampal tissue preparation}

Mice from each group were deeply anesthetized with $3 \%$ sevoflurane at $1(n=8), 4(n=6)$ and $8(n=6)$ days postsurgery. Mice were decapitated and hippocampi were isolated from the brain on ice and stored at $-80^{\circ} \mathrm{C}$ prior to use in the following experiments.

\section{Enzyme-linked immunosorbent assay (ELISA)}

The concentrations of interleukin-1 $\beta$ (IL-1 $\beta$ ) and tumor necrosis factor $\alpha$ (TNF- $\alpha$ ) were measured in hippocampal tissues on Days 1, 4, and 8 post-surgery using ELISA, following the manufacturer's instructions. The specific steps employed and description are consistent with a previous study [26]. For quantification, the concentrations of IL- $1 \beta$ and TNF- $\alpha$ were calculated based on the $450 \mathrm{~nm}$ wavelength absorbance on a spectrophotometer and expressed as $\mathrm{pg} / \mathrm{mg}$ protein.

\section{NO concentration detection}

On Day 1 post-surgery, the NO concentration in hippocampal tissues was detected using the nitrate reductase method, according to a previous report [28]. Briefly, the operation sequence complied with the manufacturer's instructions for the commercially available NO Fluorometric Assay Kit (Nanjing jiancheng biology co. LTD, Nanjing, China). The concentration of NO is presented as $\mathrm{nmol} / \mathrm{mg}$ protein and was calculated using the 550 $\mathrm{nm}$ absorbance wavelength.

\section{Western blot (WB)}

WB was performed to evaluate iNOS protein content in hippocampal tissues at $24 \mathrm{~h}$ post-surgery. Briefly, protein was extracted from the hippocampus then treated with RIPA lysis buffer (Beyotime, China) for $30 \mathrm{~min}$ and centrifuged at $14,000 \times g$ for $30 \mathrm{~min}$. Equal amounts of protein were separated by $10 \%$ sodium dodecyl sulfatepolyacrylamide gel electrophoresis and transferred to a polyvinylidene difluoride membrane (Millipore). After blocking with $5 \%$ milk in Tris buffered saline containing $0.05 \%$ Tween-20 (TBS-T) at room temperature for $1 \mathrm{~h}$, the membrane was incubated at $4{ }^{\circ} \mathrm{C}$ overnight with the primary iNOS antibody (1:500, Ab15323, Abcam, Cambridge, MA), and an anti- $\beta$-tubulin antibody (1:500, Ab6046, Abcam, Cambridge, MA) was used as a control. After washing with TBS-T and further incubation with the secondary antibody, horse radish peroxidase (HRP)conjugated anti-rabbit IgG (1:1000, A0545, Sigma-Aldrich, St. Louis, MO), at $37^{\circ} \mathrm{C}$ for $2 \mathrm{~h}$, the blots were visualized using an enhanced chemiluminescence (ECL) reagent.

\section{Oxidative stress detection}

Twenty-four hours post-surgery, the concentrations of the oxidative product malondialdehyde (MDA) and antioxidant enzyme superoxide dismutase (SOD) were measured in hippocampal tissues using appropriate kits from Nanjing Jiancheng Bioengineering Institute (A006-2, A001-3), according to the manufacturer's instructions.

\section{Immunohistochemistry (IHC)}

The microglial marker ionized calcium binding adaptor molecule 1 (IBA-1) was detected in the hippocampal cornu ammonis (CA) 1 and CA3 1 day post-surgery using IHC staining, as previously described [29, 30]. Briefly, hippocampal tissues were harvested and postfixed. Sections of 4- $\mu \mathrm{m}$ thickness were cut using a freezing microtome (Leica CM1900, Germany), collected in a 24-well plate, and rinsed. After immersing in $0.01 \mathrm{M}$ PBS containing $5 \%$ goat non-immune serum and $0.3 \%$ TritonX-100 solution at $37^{\circ} \mathrm{C}$ for $30 \mathrm{~min}$, the slices were subsequently incubated for $48 \mathrm{~h}$ at $4{ }^{\circ} \mathrm{C}$ with $2 \%$ goat serum containing the goat polyclonal primary antibody anti-IBA-1 (1:100; WAKO). The slices were washed and 
incubated in Reagents I and II from the Reagent Kit (Chemicon, Anti-Rabbit/Mouse Poly-HRP IHC Detection Kit, USA) for $30 \mathrm{~min}$ each at $37^{\circ} \mathrm{C}$. The slices were again rinsed five times, each for $5 \mathrm{~min}$ in $0.01 \mathrm{M}$ PBS-T. Finally, sections were detected using 3,3'-Diaminobenzidine (DAB) staining. A negative control was generated by replacing the primary antibody with $2 \%$ goat serum to ascertain the specificity of antibody staining. Immunoreactive products were observed and photographed with a light microscope (Leica. DMIRB, Germany) coupled with a computer assisted video camera.

\section{Tunel staining}

Tunel staining was performed on Day 1 post-surgery to detect apoptotic cells in the hippocampal CA1 and CA3. The procedure was as follows: after freezing, $50 \mu \mathrm{l}$ of freshly diluted proteinase $\mathrm{K}$ was added to the tissue at a concentration of $20 \mu \mathrm{g} / \mathrm{ml}(147 \mu \mathrm{l}$ of $10 \mathrm{mM}$ Tris- $\mathrm{HCl}$ added to $3 \mu \mathrm{l} 1 \mathrm{mg} / \mathrm{ml}$ proteinase $\mathrm{K}$ ). Digestion was performed for $15 \mathrm{~min}$ at $37^{\circ} \mathrm{C}$, followed by washing in 0.01 M PBS three times for $5 \mathrm{~min}$ each. Then, the slices were rinsed using $0.1 \%$ diethyl pyrocarbonate (DEPC) water at room temperature for $30 \mathrm{~min}$. PBS $(0.01 \mathrm{M})$ was used to wash the sections three times for 5 min each. Redundant fluid was removed from the slices. Solution I and Solution II were added (1:9 volume ratio, prepared on PE gloves on ice, well distributed). The Tunel reaction mixture was prepared. Subsequently, freshly prepared 3\% $\mathrm{H}_{2} \mathrm{O}_{2}$-methanol was added and incubated with the sections at room temperature for $15 \mathrm{~min}$, followed by washing with $0.01 \mathrm{M}$ PBS three times for $5 \mathrm{~min}$ each. Confining liquid $(50 \mu \mathrm{l} 5 \%$ bovine serum albumin) was added and sections were incubated for $30 \mathrm{~min}$ at $37^{\circ} \mathrm{C}$. Then, the confining liquid was removed without washing. Transforming agent-AP $(50 \mu \mathrm{l})$ was added to each slice. Then, the slices were placed in the wet box and incubated for $40 \mathrm{~min}$ at $37^{\circ} \mathrm{C}$ followed by washing with $0.01 \mathrm{M}$ PBS three times for $5 \mathrm{~min}$ each. TVBT was used for color production at room temperature. A general light microscope was used to observe the color for 5-10 min. Distilled water was used to stop the color reaction. The sections were washed under flowing water for 10 min to clean the NBT grains. Conventional dehydration and mounting were performed. A light microscope was used to observe the result of Tunel staining. In the negative control group, Solution II was added without Solution 1 . The sample slices were covered with a plastic cap. Then, the samples were placed into a wet box, labeled for $1 \mathrm{~h}$ at $37^{\circ} \mathrm{C}$ and then overnight at $4{ }^{\circ} \mathrm{C}$ for more than $20 \mathrm{~h}$. Then, samples were washed with $0.01 \mathrm{M}$ PBS three times for $3 \mathrm{~min}$ each. In the positive control group, Dnase 1 was added and incubated at room temperature for $10 \mathrm{~min}$. Lastly, the numbers of cells undergoing apoptosis and the morphological features were observed.

\section{Statistical analysis}

Data were analyzed using SPSS 17.0 software and expressed as the mean \pm standard deviation (SD). Comparisons between multiple groups were performed with one-way analysis of variance (ANOVA) or a two-way ANOVA with a Bonferroni's multiple comparisons posthoc test where appropriate. Data from the MWM test were analyzed using a two-way ANOVA (treatment $\times$ trial time) with repeated measures (trial days). Differences were considered to be statistically significant at $P<0.05$.

\section{Results}

Exogenous $\mathrm{H}_{2} \mathrm{~S}$ reverses impaired cognitive function without altering the locomotor dysfunction caused by surgical injury, and is similar to L-NAME

In our study, cognitive performance was assessed using the OFT and MWM. In the OFT test, the total moving distance and moving duration data showed no visible difference in locomotor activity between the groups (Fig. 1a, b). MWM outcomes showed that surgery reduced spatial learning, with a longer escape latency and path length and decreased memory ability with a reduced percent time and length in the target quadrant when compared to the sham group. Compared with the sham group, the exogenous $\mathrm{H}_{2} \mathrm{~S}$ donor GYY4137 significantly improved spatial learning and memory capacity, and, compared with the surgery group, GYY4137 could partially reverse the effects of surgical injury. The NOS inhibitor L-NAME alone did not influence learning and memory performance compared with the sham group, but compared with the surgery group it also partially reversed surgery-induced learning and memory decline. The effect was similar to GYY4137. Meanwhile, the combination of GYY4137 and L-NAME did not exert additional advantageous effects compared with GYY4137 or L-NAME alone (Fig. 1c-f). These findings suggest that the cognitive deficits in spatial learning and memory were not owing to a reduction in spontaneous locomotor activity.

\section{Exogenous $\mathrm{H}_{2} \mathrm{~S}$ reverses surgery-induced neuroinflammation on day 1 and day 4 post-surgery, but not on day 8 post-surgery, which may be related to the reduction in NO and iNOS concentrations in the hippocampus, and the effect is similar to L- NAME}

Surgery induced marked neuroinflammation in the hippocampus with increased concentrations of IL- $1 \beta$ and TNF- $\alpha$ noted, compared to the sham group. The highest concentration appeared on Day 1 postsurgery, and the neuroinflammation was alleviated by Day 4 post-surgery. The neuroinflammation status was substantially restored on Day 8 post-surgery. No significant difference was noted between groups. Both 


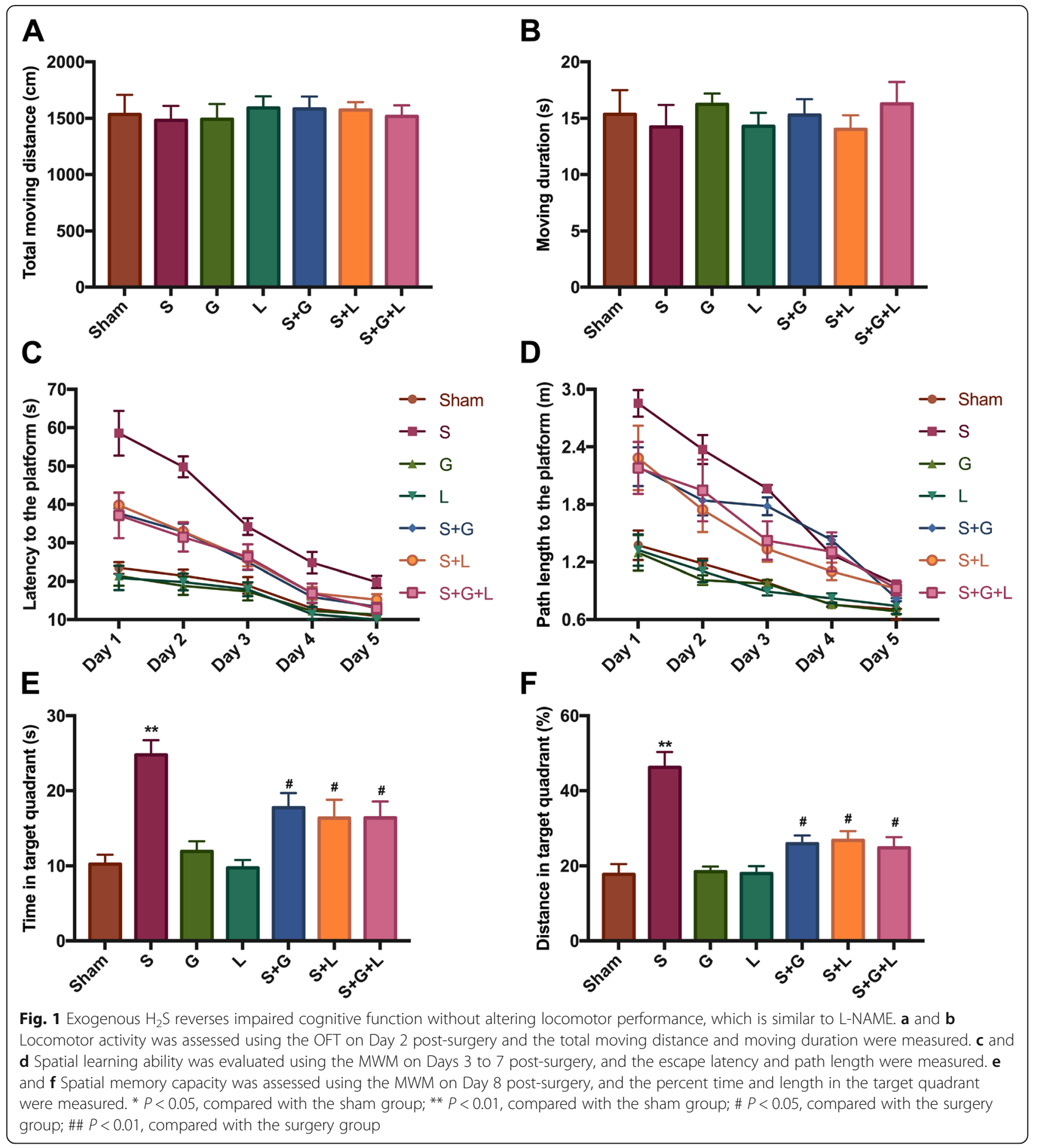

GYY4137 and L-NAME could inhibit the surgeryinduced neuroinflammation, and the combination of GYY4137 and L-NAME did not produce a better effect compared with GYY4137 or L-NAME alone (Fig. 2a, b). Furthermore, we detected the NO and iNOS in the hippocampus at $24 \mathrm{~h}$ post-surgery, and the results were consistent with those for IL-1 $\beta$ and TNF- $\alpha$ (Fig. 2c, d).
Exogenous $\mathrm{H}_{2} \mathrm{~S}$ reverses surgery-induced oxidative stress, microglial activation, and cell apoptosis in the hippocampus, which is similar to L-NAME

Twenty-four hours post-surgery, oxidative stress, microglial activation, and cell apoptosis were assessed using MDA and SOD kits, IHC, and Tunel staining, respectively. The results show that surgery induced an oxidative stress reaction in the hippocampus, with increased 


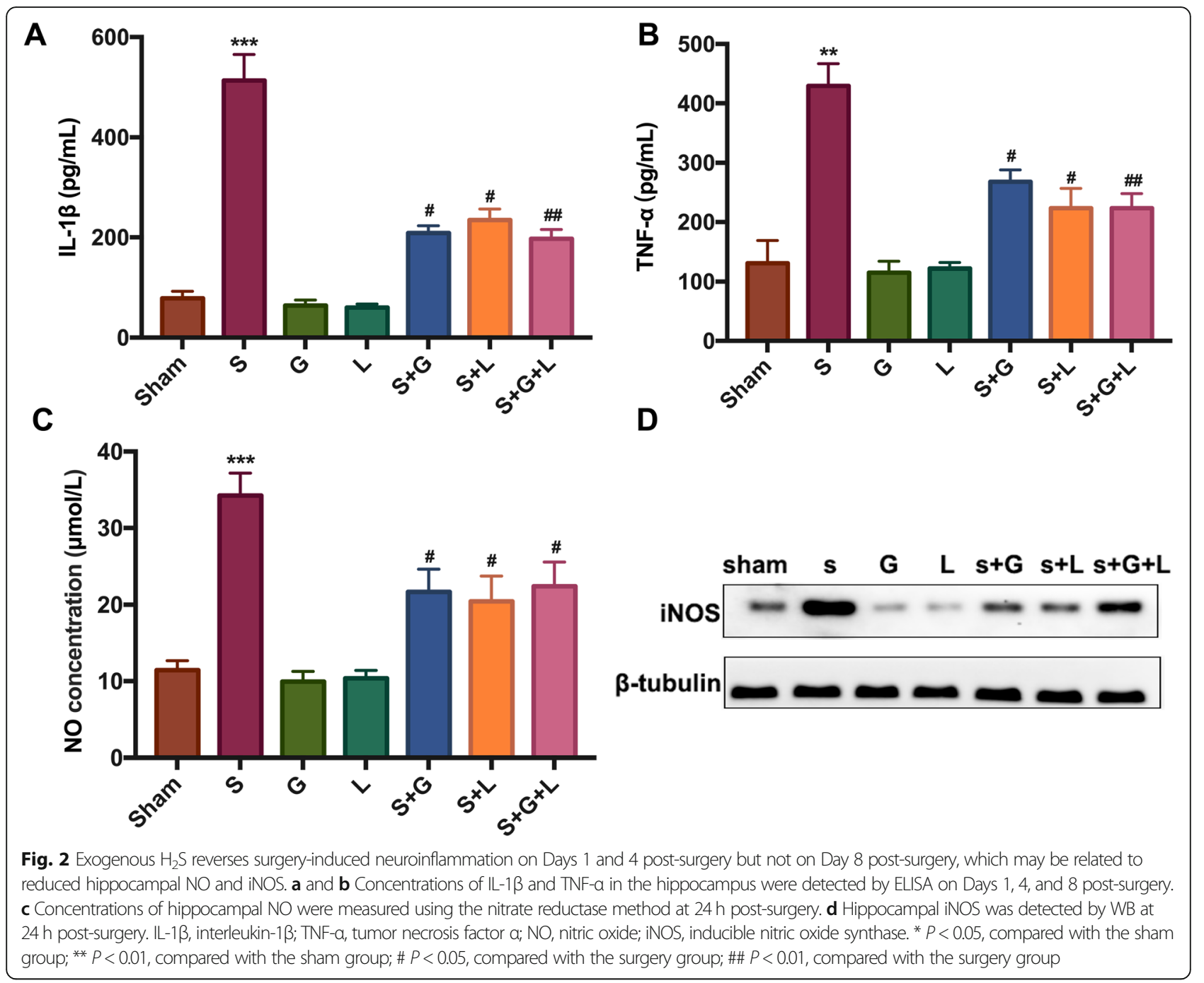

production of MDA and decreased production of SOD noted. Treatment with GYY4137 or L-NAME mitigated the degree of oxidative stress. However, GYY4137 or L-NAME alone had no effect. Moreover, the combination of GYY4137 and L-NAME had no additional advantage, compared with GYY4137 or L-NAME alone (Fig. 3a). Additionally, more microglial and apoptotic cells were found in the surgery group compared to the sham group. Mice treated with GYY4137 or L-NAME showed attenuation of microglial activation and cell apoptosis in the hippocampal CA1 and CA3. There were no marked differences between the sham group and GYY4137 group, or between the sham group and LNAME group. Moreover, the combination of GYY4137 and L-NAME was not significantly better than GYY4137 or LNAME alone (Fig. 3b, c). These data indicate that the protective effects of GYY4137 are similar to L-NAME.

\section{Discussion}

In this study, we identified that the $\mathrm{H}_{2} \mathrm{~S}$ donor GYY4137 plays a protective role in surgery-induced neuroinflammatory cognitive disorder in adult mice by suppressing $\mathrm{NO}$ and iNOS in the hippocampus. Right carotid artery exposure surgery caused an increase in the pro-inflammatory cytokines IL-1 $\beta$ and TNF- $\alpha$ in the hippocampus, and impaired spatial learning and memory ability, but not locomotor activity. The increase in hippocampal NO and iNOS, accumulation of the oxidative product MDA, and reduction in the antioxidant enzyme SOD in the hippocampus, microglial activation, and cell apoptosis in the hippocampal CA1 and CA3 were all remedied by the $\mathrm{H}_{2} \mathrm{~S}$ donor GYY4137 or iNOS inhibitor LNAME. Moreover, the combined application of GYY4137 and L-NAME was not superior to their individual application, and the effect of GYY4137 was similar to L-NAME.

Clinical data have shown that anesthesia and surgical trauma are the main causes of POCD, which may increase morbidity and mortality [3]. In addition, surgeryinduced neuroinflammation in the hippocampus is closely related to POCD [26]. Diverse surgical operations performed on animals have been adopted to mimic POCD [8, 31, 32]. Wang et al. demonstrated that right 


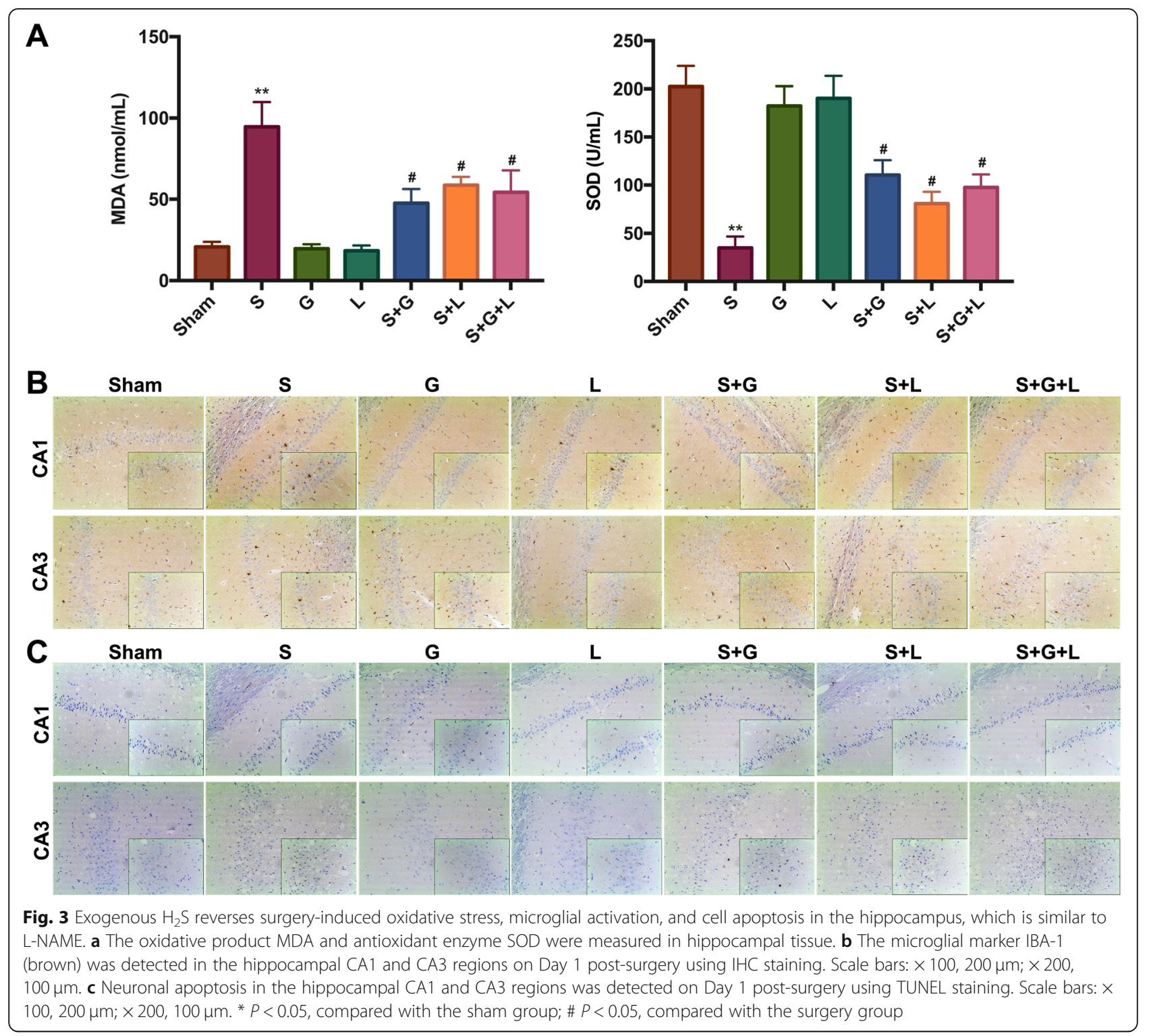

carotid artery exposure induced apparent cognitive impairment in spatial learning and memory, as detected using the MWM test, and reduced freezing time in the FCS experiment, but did not impair locomotor activity in the OFT or attenuate the increase in the proinflammatory cytokines IL-1 $\beta$, IL-6, and TNF- $\alpha$ in the hippocampus on Day 4 post-surgery in aged C57BL/6 J male mice [26]. Another previous study also reported that carotid artery exposure induced POCD and a neuroinflammatory response, as well as reduced neurogenesis in adult C57BL/6 J mice. Our study used adult C57BL/6 J mice and mimicked the surgery model used by Wang et al. to obtain consistent results. Notably, the $\mathrm{H}_{2} \mathrm{~S}$ donor GYY4137 and iNOS inhibitor L-NAME effectively ameliorated the surgery-induced neuroinflammation and cognitive impairment.
$\mathrm{NO}$ is generated excessively following inflammatory stimulation and may induce neurodegeneration and accelerate cognitive decline and dementia [33]. NO is produced by NOS, and iNOS expression is not restricted to specific cells and represents a major pro-inflammatory and destructive mediator in inflammatory disease [20]. The suppression of iNOS plays a neuroprotective role, including reducing traumatic brain injury-induced synaptic plasticity and cognitive function deficits in male C57BL/6 J mice [21]. In our work, surgery induced distinct neuroinflammation in the hippocampus and stimulated a high level of iNOS expression, which promoted $\mathrm{NO}$ oversynthesis in the hippocampus. However, treatment with GYY4137 or L-NAME reversed the inflammation-induced overproduction of iNOS and NO in the hippocampus. The combined application of 
GYY4137 and L-NAME did not produce additional beneficial effects, and the effect of GYY4137 was similar to L-NAME. These results indicate that exogenous $\mathrm{H}_{2} \mathrm{~S}$ alleviates surgery-induced neuroinflammatory cognitive impairment in adult mice through the inhibition of $\mathrm{NO}$ signaling in the hippocampus. Endogenous and exogenous $\mathrm{H}_{2} \mathrm{~S}$ has been shown to exert anti-inflammatory effects that induce recovery from inflammatory disease, such as colitis [34]. A previous study suggested that exogenous $\mathrm{H}_{2} \mathrm{~S}$ relieves spatial memory impairments in $\mathrm{AD}$ [35]. Exogenous $\mathrm{H}_{2} \mathrm{~S}$ also been demonstrated to have neuroprotective effects against brain edema, neurobehavioral function, including learning and memory, and neuronal cell death following subarachnoid hemorrhage [36]. Furthermore, $\mathrm{H}_{2} \mathrm{~S}$ can regulate the release of $\mathrm{NO}$, and its interaction with $\mathrm{NO}$ in the hippocampus can alleviate brain dysfunction [22-24]. Here, we are the first to demonstrate that exogenous $\mathrm{H}_{2} \mathrm{~S}$ ameliorates spatial learning and memory deficits in a surgery-induced POCD mouse model by inhibiting $\mathrm{NO}$ and reducing iNOS levels in the hippocampus.

In the current study, we also showed that exogenous $\mathrm{H}_{2} \mathrm{~S}$ reverses surgery-induced oxidative stress, microglial activation, and cell apoptosis in the hippocampus, which is similar to the effects of L-NAME. A previous study suggested that oxidative stress is highly connected with reduced cognition, including declined psychomotor speed, mental flexibility, and attention in patients with type 2 diabetes [37]. Oxidative damage in the hippocampus is also implicated in chemotherapy-induced cognitive impairment [38]. Besides, oxidative stress could mediate inflammation and apoptosis in hippocampus, which promote hippocampal lesions [39]. Importantly, microglial activation is a hallmark of the onset of neuroinflammation, and contributes to collateral brain injury [40]. Microglial activation plays a pivotal pathogenic role in the initiation of cognitive disorder-related illness, such as $\mathrm{AD}$ [41]. Interestingly, the present evidence indicates that autophagy, apoptosis, inflammation, and oxidative stress play a possible role in the pathogenesis of neurodegenerative disorders that are characterized by a loss of neuronal function [42]. Additionally, NO induces neuronal modifications, for example, microglial activation, neuronal cell apoptosis, and oxidative stress, and high concentrations of $\mathrm{NO}$ are harmful to cognitive performance. The bioavailability of NO is recognized as a predictive risk marker for $\mathrm{AD}$ and early POCD [17-19]. Therefore, the surgery-induced inflammatory response, which induces high levels of iNOS expression in the hippocampus, then causes NO overproduction in the hippocampus followed by the promotion of oxidative stress, microglial activation, neuronal cell apoptosis, and hippocampal lesions eventually induce the development of POCD.
Treatment with GYY4137 or L-NAME can partially restore impaired cognitive function.

\section{Conclusion}

In summary, we found that the long-acting hydrogen sulfide donor GYY4137 had an ability to reversed the cognitive deficits and inflammation caused by carotid artery exposure surgery. This implies that NO signaling pathways might participate in this process. The results indicate that exogenous $\mathrm{H}_{2} \mathrm{~S}$ may be a promising therapy for POCD.

\section{Abbreviations}

AD: Alzheimer's disease; ANOVA: One-way analysis of variance; CA: Cornu ammonis; DAB: Diaminobenzidine; DEPC: Diethyl pyrocarbonate;

ECL: Enhanced chemiluminescence; ELISA: Enzyme-linked immunosorbent assay; eNOS: Endothelial NOS; $\mathrm{H}_{2} \mathrm{~S}$ : Hydrogen sulfide; HRP: Horse radish peroxidase; IBA-1: Ionized calcium binding adaptor molecule 1; IHC: Immunohistochemistry; IL-1ß: Interleukin-1 $\beta$; iNOS: Inducible NOS; LNAME: L-nitro-arginine methyl ester; MDA: Malondialdehyde; MWM: Morris water maze; nNOS: Neuronal NOS; NO: Nitric oxide; NOS: Nitric oxide synthases; PBS: Phosphate buffered saline; POCD: Post-operative cognitive dysfunction; SD: Standard deviation; SOD: Superoxide dismutase; TBS-T: Tris buffered saline containing 0.05\% Tween-20; TNF-a: Tumor necrosis factor a WB: Western blot

\section{Acknowledgements \\ Not applicable.}

Authors' contributions

LY and CL conceived and designed study. LY and SG performed research, analyzed data and wrote the manuscript. $C L$ reviewed and revised the manuscript. All authors reviewed and approved the final manuscript.

\section{Funding}

Not applicable.

\section{Availability of data and materials}

The datasets used and/or analysed during the current study are available from the corresponding author on reasonable request.

\section{Ethics approval and consent to participate}

The mice were treated according to the ethical guidelines of the Animal Experiment Center of the Institute of Radiation Medicine of the Chinese Academy of Medical Science, and the Animal Studies Committee of the Animal Experiment Center of the Institute of Radiation Medicine of the

Chinese Academy of Medical Science approved the experimental protocol.

\section{Consent for publication}

Not applicable.

\section{Competing interests}

The authors declare that they have no competing interests.

Received: 29 August 2019 Accepted: 30 December 2019

Published online: 09 January 2020

References

1. Fan D, Li J, Zheng B, Hua L, Zuo Z. Enriched environment attenuates surgery-induced impairment of learning, memory, and neurogenesis possibly by preserving BDNF expression. Mol Neurobiol. 2016;53:344-54.

2. $\mathrm{Ni} \mathrm{C}, \mathrm{Xu} T$, Li N, Tian Y, Han Y, Xue Q, et al. Cerebral oxygen saturation after multiple perioperative influential factors predicts the occurrence of postoperative cognitive dysfunction. BMC Anesthesiol. 2015;15:156.

3. Monk TG, Weldon BC, Garvan CW, Dede DE, van der Aa MT, Heilman KM, et al. Predictors of cognitive dysfunction after major noncardiac surgery. Anesthesiology. 2008;108:18-30. 
4. Steinmetz J, Christensen KB, Lund T, Lohse N, Rasmussen LS. Long-term consequences of postoperative cognitive dysfunction. Anesthesiology. 2009; 110:548-55.

5. Steinmetz J, Siersma V, Kessing LV, Rasmussen LS. Is postoperative cognitive dysfunction a risk factor for dementia? A cohort follow-up study. $\mathrm{Br} J$ Anaesth. 2013:110(Suppl 1):i92-7.

6. Tuman KJ, McCarthy RJ, Najafi H, Ivankovich AD. Differential effects of advanced age on neurologic and cardiac risks of coronary artery operations. J Thorac Cardiovasc Surg. 1992;104:1510-7.

7. Wang W, Wang $Y$, Wu H, Lei L, Xu S, Shen $X$, et al. Postoperative cognitive dysfunction: current developments in mechanism and prevention. Med Sc Monit. 2014;20:1908-12.

8. Xu Z, Dong Y, Wang H, Culley DJ, Marcantonio ER, Crosby G, et al. Peripheral surgical wounding and age-dependent neuroinflammation in mice. PLoS One. 2014:9:e96752.

9. Xu Z, Dong Y, Wang H, Culley DJ, Marcantonio ER, Crosby G, et al. Agedependent postoperative cognitive impairment and Alzheimer-related neuropathology in mice. Sci Rep. 2014;4:3766.

10. Driscoll I, Hamilton DA, Petropoulos H, Yeo RA, Brooks WM, Baumgartne RN, et al. The aging hippocampus: cognitive, biochemical and structural findings. Cereb Cortex. 2003;13:1344-51

11. Hovens IB, Schoemaker RG, van der Zee EA, Absalom AR, Heineman E, van Leeuwen BL. Postoperative cognitive dysfunction: involvement of neuroinflammation and neuronal functioning. Brain Behav Immun. 2014;38:202-10.

12. Hovens IB, van Leeuwen BL, Nyakas C, Heineman E, van der Zee EA Schoemaker RG. Postoperative cognitive dysfunction and microglial activation in associated brain regions in old rats. Neurobiol Learn Mem. 2015;118:74-9

13. Zhang J, Tan H, Jiang W, Zuo Z. The choice of general anesthetics may not affect neuroinflammation and impairment of learning and memory after surgery in elderly rats. J Neurolmmune Pharmacol. 2015;10:179-89.

14. Cibelli M, Fidalgo AR, Terrando N, Ma D, Monaco C, Feldmann M, et al. Role of interleukin-1 beta in postoperative cognitive dysfunction. Ann Neurol. 2010;68:360-8.

15. Cao L, Li L, Lin D, Zuo Z. Isoflurane induces learning impairment that is mediated by interleukin $1 \beta$ in rodents. PLoS One. 2012;7:e51431.

16. Liu C, Liang MC, Soong TW. Nitric oxide, iron and neurodegeneration. Front Neurosci. 2019;13:114

17. Shi Q, Liu X, Wang N, Zheng X, Ran J, Liu Z, et al. 1400W ameliorates acute hypobaric hypoxia/reoxygenation-induced cognitive deficits by suppressing the induction of inducible nitric oxide synthase in rat cerebral cortex microglia. Behav Brain Res. 2017;319:188-99.

18. Venturelli M, Pedrinolla A, Boscolo Galazzo I, Fonte C, Smania N, Tamburin S, et al. Impact of nitric oxide bioavailability on the progressive cerebral and peripheral circulatory impairments during aging and Alzheimer's disease. Front Physiol. 2018;9:169.

19. Iohom G, Szarvas S, Larney V, O'Brien J, Buckley E, Butler M, et al. Perioperative plasma concentrations of stable nitric oxide products are predictive of cognitive dysfunction after laparoscopic cholecystectomy. Anesth Analg. 2004;99:1245-52 table of contents.

20. Leonidou A, Lepetsos P, Mintzas M, Kenanidis E, Macheras G, Tzetis M, et al. Inducible nitric oxide synthase as a target for osteoarthritis treatment. Expert Opin Ther Targets. 2018:22:299-318.

21. Wang B, Han S. Inhibition of inducible nitric oxide synthase attenuates deficits in synaptic plasticity and brain functions following traumatic brain injury. Cerebellum. 2018;17:477-84.

22. Askari H, Abazari MF, Ghoraeian P, Torabinejad S, Nouri Aleagha M, Mirfallah Nassiri R, et al. Ameliorative effects of hydrogen sulfide (NaHS) on chronic kidney disease-induced brain dysfunction in rats: implication on role of nitric oxide (NO) signaling. Metab Brain Dis. 2018;33:1945-54.

23. Cao X, Ding L, Xie ZZ, Yang Y, Whiteman M, Moore PK, et al. A review of hydrogen sulfide synthesis, metabolism, and measurement: is modulation of hydrogen sulfide a novel therapeutic for cancer. Antioxid Redox Signal. 2019:31:1-38.

24. Merighi S, Gessi S, Varani K, Fazzi D, Borea PA. Hydrogen sulfide modulates the release of nitric oxide and VEGF in human keratinocytes. Pharmacol Res. 2012;66:428-36.

25. Drucker NA, Jensen AR, Te Winkel JP, Markel TA. Hydrogen sulfide dono GYY4137 acts through endothelial nitric oxide to protect intestine in murine models of necrotizing Enterocolitis and intestinal ischemia. J Surg Res. 2019; 234:294-302.
26. Wang $W$, Zhang $X Y$, Feng $Z G$, Wang DX, Zhang $H$, Sui $B$, et al. Overexpression of phosphodiesterase-4 subtypes involved in surgeryinduced neuroinflammation and cognitive dysfunction in mice. Brain Res Bull. 2017:130:274-82.

27. Park SS, Stranahan AM, Chadwick W, Zhou Y, Wang L, Martin B, et al. Cortical gene transcription response patterns to water maze training in aged mice. BMC Neurosci. 2011;12:63.

28. Nussler AK, Glanemann M, Schirmeier A, Liu L, Nüssler NC. Fluorometric measurement of nitrite/nitrate by 2,3-diaminonaphthalene. Nat Protoc. 2006;1:2223-6.

29. Zhao WX, Zhang JH, Cao JB, Wang W, Wang DX, Zhang XY, et al. Acetaminophen attenuates lipopolysaccharide-induced cognitive impairment through antioxidant activity. J Neuroinflammation. 2017;14:17.

30. Walker DG, Lue LF, Beach TG, Tooyama I. Microglial phenotyping in neurodegenerative disease brains: identification of reactive microglia with an antibody to variant of CD105/Endoglin. Cells. 2019;8. https://doi.org/10. 3390/cells8070766.

31. Tian Y, Guo S, Zhang Y, Xu Y, Zhao P, Zhao X. Effects of hydrogen-rich saline on hepatectomy-induced postoperative cognitive dysfunction in old mice. Mol Neurobiol. 2017:54:2579-84.

32. Wang P, Cao J, Liu N, Ma L, Zhou X, Zhang H, et al. Protective effects of Edaravone in adult rats with surgery and lipopolysaccharide administrationinduced cognitive function impairment. PLoS One. 2016;11:e0153708.

33. Stephan B, Harrison SL, Keage H, Babateen A, Robinson L, Siervo M. Cardiovascular disease, the nitric oxide pathway and risk of cognitive impairment and dementia. Curr Cardiol Rep. 2017:19:87.

34. Wallace $J$, Vong L, McKnight W, Dicay M, Martin GR. Endogenous and exogenous hydrogen sulfide promotes resolution of colitis in rats. Gastroenterology. 2009;137:569-578.e1

35. Yang YJ, Zhao Y, Yu B, Xu GG, Wang W, Zhan JQ, et al. GluN2B-containing NMDA receptors contribute to the beneficial effects of hydrogen sulfide on cognitive and synaptic plasticity deficits in APP/PS1 transgenic mice. Neuroscience. 2016:335:170-83.

36. Li T, Liu H, Xue H, Zhang J, Han X, Yan S, et al. Neuroprotective effects of hydrogen sulfide against early brain injury and secondary cognitive deficits following subarachnoid hemorrhage. Brain Pathol. 2017;27:51-63.

37. Groeneveld ON, van den Berg $E$, Johansen $O E$, et al. Oxidative stress and endothelial dysfunction are associated with reduced cognition in type 2 diabetes. Diab Vasc Dis Res. 2019;16:577-81.

38. Bagnall-Moreau C, Chaudhry S, Salas-Ramirez K, Ahles T, Hubbard K. Chemotherapy-Induced Cognitive Impairment Is Associated with Increased Inflammation and Oxidative Damage in the Hippocampus. Mol Neurobiol. 2019;56:7159-72.

39. Cao Z, Wang P, Gao X, Shao B, Zhao S, Li Y. Lycopene attenuates aluminum-induced hippocampal lesions by inhibiting oxidative stressmediated inflammation and apoptosis in the rat. J Inorg Biochem. 2019;193: $143-51$

40. Xu P, Zhang X, Liu Q, Xie Y, Shi X, Chen J, et al. Microglial TREM-1 receptor mediates neuroinflammatory injury via interaction with SYK in experimental ischemic stroke. Cell Death Dis. 2019;10:555.

41. Gao G, Zhao S, Xia X, Li C, Li C, Ji C, et al. Glutaminase C regulates microglial activation and pro-inflammatory exosome release: relevance to the pathogenesis of Alzheimer's disease. Front Cell Neurosci. 2019;13:264.

42. Pourhanifeh MH, Shafabakhsh R, Reiter RJ, Asemi Z. The Effect of Resveratrol on Neurodegenerative Disorders: Possible Protective Actions Against Autophagy, Apoptosis, Inflammation and Oxidative Stress. Curr Pharm Des. 2019:25:2178-91.

\section{Publisher's Note}

Springer Nature remains neutral with regard to jurisdictional claims in published maps and institutional affiliations. 